\title{
Automation and productivity in the clinical laboratory: experience of a tertiary healthcare facility
}

Chin Pin $\underline{\mathrm{YeO}}^{1}$, MBBS, FRCPA, Wai Yoong $\underline{\mathrm{Ng}}^{1}$, PhD, CSci

\begin{abstract}
Clinical laboratories for in vitro diagnostics are facing pressure to preserve cost control while providing better services through new initiatives. Laboratory automation is a partial answer to this problem, having come a long way from the early days of clinical laboratory testing. The journey and implementation of automation in the Singapore General Hospital's Clinical Biochemistry Laboratory has allowed for sustained performance in the light of increasing workload and service commitments amid an evolving healthcare environment. Key to realising predicted outcomes is the optimisation of workflow processes, reduction of errors, and spatial placement of specimen reception and analytical areas. This paper gives an overview of our experience with automation in the clinical laboratory and its subsequent impact on service standards.
\end{abstract}

Keywords: clinical biochemistry laboratory, laboratory automation, lean, productivity

\section{INTRODUCTION}

The clinical laboratory for in vitro diagnostics is facing pressure these days to preserve cost control while constantly being nudged to provide better services through new initiatives. Another vital challenge for diagnostics testing in the future is the shrinking workforce, ${ }^{(1-4)}$ which is attributed to increased competition for a finite talent pool and an ageing population. Technological advances in laboratory testing, complemented by a shift in laboratory management and operations, may be the answer.

\section{What is laboratory automation?}

The layman's perspective on laboratory automation may be entirely different from that of a professional. To the former, it could mean the prospect of replacing humans with robots. To the laboratorian, it means the re-engineering of processes - from tedious, repetitive manual labour to robotics - so that skilled manpower can perform more value-added work such as quality monitoring, evaluation, and setting up new tests and collaborative clinical research.

\section{How does laboratory automation fit into the current landscape?}

The early predictions of an ageing population, first seen in Western societies, now appear to have reached the shores of the East, and Singapore is not spared. It has been projected that by 2030, Singapore will have 900,000 seniors (aged above 65 years), a doubling of the 2015 projected figure of 440,000 . This gives a ratio of one in four Singaporeans aged above 65 years, as compared to one in eight in 2015. ${ }^{(5)}$ An ageing population also means a greater demand for hospitals, both tertiary and community, to look after the elderly. Indeed, to cater to this rising trend, the healthcare expansion plans of the Ministry of Health, Singapore, aim to roll out regional and community hospitals every year until 2020.
Dr Masahide Sasaki of Kochi Medical School in Japan first expounded on the term 'total laboratory automation' (TLA) in the 1980s. He described TLA as a setup of analytical instruments connected via a specimen transport track system. ${ }^{(6,7)}$ Laboratory automation, apart from its prediction of better turnaround time (TAT) through increased efficiency, served as the panacea to manpower issues and the purposeful answer to a looming manpower shortage predicted in the United States (US) due to the ageing baby boomers (those born after World War II). In this article, we provide insights on our automation journey in the clinical laboratory and its subsequent impact on service standards.

\section{LOCAL DEVELOPMENTS}

The Singapore General Hospital's Clinical Biochemistry Laboratory had its beginnings in the early 1900s when the first government pathological service was founded. Advances in technology and the arrival of the computer age pushed the frontiers of clinical laboratory testing to phenomenal heights. The laboratory started introducing a wide repertoire of tests, electronic result reporting and linkages to healthcare clinical reporting systems, among others. In 1969, our institution purchased its first single-channel continuous flow Technicon AutoAnalyzer, which has been widely cited in the literature as the first 'true' automated instrument for the clinical laboratory. It enabled blood analysis from beginning to the end with little human intervention. The following year, two additional instruments were acquired by our institution. The ensuing years saw such rapid growth with the introduction of other single- and multi-channel instruments that by the end of the 1970 s, approximately $80 \%$ of clinical biochemistry work was associated with automated analysers. In the early 1980s, random-access analysers such as the Beckman Astra-8 were introduced. ${ }^{(8)}$ Dr Sasaki's concept of TLA, although yet to be fully embraced by our laboratory, was always up for consideration. 
With advancements in the TLA concept and its key components being made available in the larger commercial offerings of the late 1990s, our laboratory embraced the new advances with a flurry of procurements. At the height of its activity, it saw the installation of state-of-the-art chemistry and immunoassay analysers from up to eight different manufacturers. In anticipation of a projected increase in workload (5.5 million investigations in 2006) and to achieve an improved, sustainable and predictable TAT of test results for STAT and urgent cases in our 24/7 Clinical Biochemistry Laboratory, our clinical laboratory streamlined and standardised work processes through the consolidation of analytical platforms, and formally introduced a 'true' TLA in 2007 with the placement of connected analysers (both chemistry and immunoassay models), pre- and postanalytical specimen processing modules and an intelligent track system that auto-routes test orders and specimens (Fig. 1).

We gained important insights from the experience of accommodating pieces of hardware into existing laboratory space that was not purpose-designed. Firstly, we learnt that staff buy-in is vital. Initial unfamiliarity among the staff with handling TLA mechanics and psychological resistance to the loss of hands-on control of specimen routing contributed to teething issues, and we realised that more pre-installation efforts in terms of training and engagement would have made it easier for the staff to embrace a new system. Secondly, we recognised that putting in place a lean workflow prior to automation allowed us to reap the full benefits of TLA. Thirdly, we realised that improvements in infrastructure and facilities, such as power, water supply lines and data points, should be carefully considered before the commencement of renovations. Finally, we discovered that space constraints may preclude certain TLA configurations or the desired number of analysers. Despite the challenges and constraints faced, the promise of laboratory automation to stabilise and predict TAT did materialise when we completed the installation. Over time, our staff also fully appreciated and benefited from the standardised workflow for the majority of specimens going through the busy laboratory.

During the period of laboratory automation, there was a fortuitous opportunity that same year in the form of a new diagnostic facility, which was to be built 4-6 years from then. The clinical biochemistry laboratory was to be relocated. It has been repeatedly pointed out that laboratory automation cannot yield improvements against a backdrop of inefficient work processes. Therefore, design considerations were a top priority during the planning of this purpose-built facility. The specimen reception facility, which was designed to streamline specimen processing, assumed a central position. This stance - taken against the background of our laboratory performing $70 \%$ of the pathology investigations - was imperative to ensure that specimens would be given prompt attention on arrival at the laboratory.

In 2011, we performed an analysis of specimen arrival patterns to set the stage for synergistic work processes. There were typically three similar arrival peaks each day between 5.30 am and 6.00 pm (Fig. 2). With over $76 \%$ of specimens arriving by pneumatic tube system and with the bulk of these $(75 \%)$ remaining on the proposed Level 8 activity area (biochemistry laboratory, haematology laboratory and central reception), it was a conjecture that the central specimen reception area should be situated near both the biochemistry and haematology priority testing areas. In 2013, we finally moved into the new facility, and the initial teething issues were managed. The specimen arrival and laboratory automation systems were in close proximity to each other. In tandem, an end-to-end solution that directly linked physician test orders (through the Computerised Physician Order Entry or CPOE) with the testing platforms enabled a quantum jump in service standards. ${ }^{(9)}$ Direct CPOE-ID labels read by the analysers made possible a seamless specimen journey from ordering to routing, arrival, analyser, analysis and results. Improvements in infrastructure support included an enhanced pneumatic tube system. Consequently, turnaround for STAT and urgent test orders can be completed within 45 minutes.

\section{PRODUCTIVITY AND MANPOWER}

Efficiency and effectiveness closely shape the productivity of the clinical biochemistry laboratory. For tangible benefits, laboratorywide practices need to be implemented. The prime goal at the time of exploiting laboratory automation was to increase efficiency as well as sustain and, hopefully, improve service performance both in terms of TAT for critical tests and our ability to accommodate a wider test repertoire on a 24/7 basis. For a measure of contribution, various productivity metrics have been proposed: service (e.g. cost, TAT); staffing (e.g. maintaining the full-time equivalent [FTE] number despite workload increases); and resource (e.g. throughput, capacity). Additionally, clinical laboratory productivity is synonymous with clinical quality. ${ }^{(10-12)}$ As early as 1984, studies have been conducted to benchmark clinical laboratory productivity in areas such as service performance, labour savings and tenable improvements. ${ }^{(13-16)}$

One key performance indicator is the TAT. Our initial performance after relocation in 2013 showed an expected dip, but recovery to pre-relocation levels was remarkably swift (within two months). Performance was up to par and, of note, consistently high (to this day), thereby reaffirming the promise of laboratory automation. Occasionally, over $90 \%$ of our completions are within 40 minutes; such a TAT is widely appreciated by the physicians on campus (Fig. 3). Less appreciated, perhaps, is the fact that automation does not guarantee good results if a poor workflow process is in place. The real benefits of laboratory automation must be in tandem with lean workflow processes and efficient specimen delivery. We were fortunate to have the opportunity to design a clinical laboratory facility in a way that incorporated the lessons learnt prior to 2013. The winning point of laboratory automation is its ability to demonstrate consistent and predictable performance over time; typically, for key critical analyses, it is preferable for TAT to be short. In our setup, incremental improvement in TAT has indeed been achieved, yet it can be argued that consistent and high performance in TAT, with accompanying improvements in workflow efficiencies and manpower productivity, is in itself the real prize. It has been recognised that efficient laboratories that turn to laboratory 


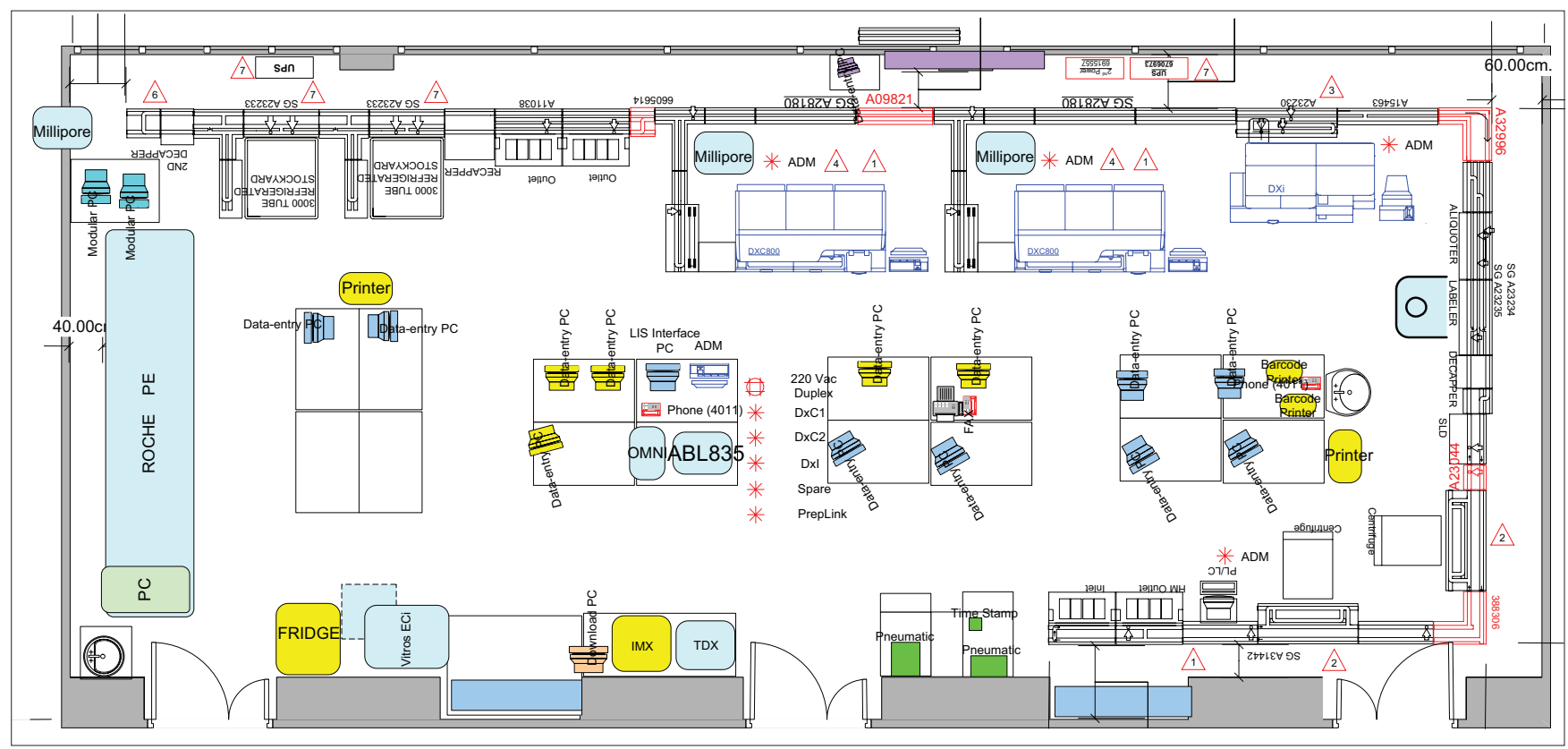

Fig. 1 Diagram of the laboratory automation system shows the schematics of instrument layout with specimen track and connections in the 24/7 Singapore General Hospital Clinical Biochemistry Laboratory in 2007.

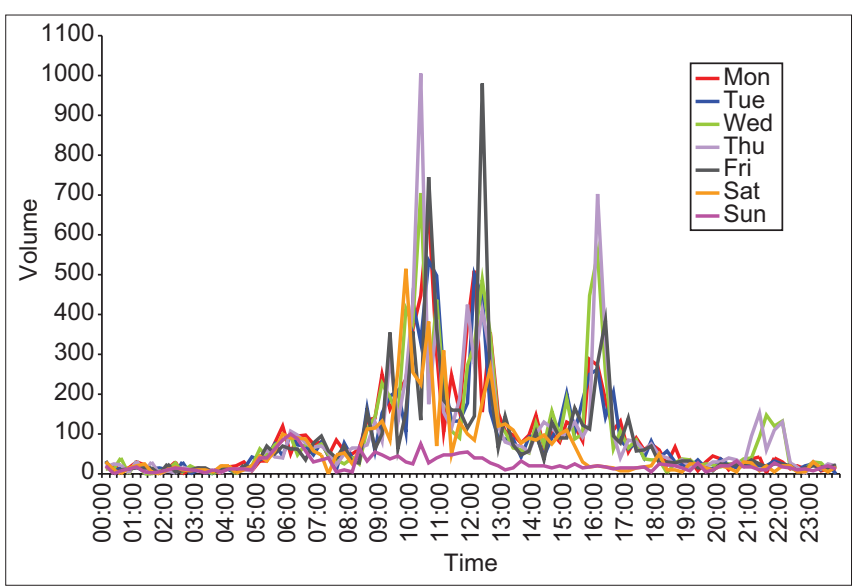

Fig. 2 Graph shows the 24-hour specimen arrival pattern for pathologica investigations (2011 SGH Specimen Management Study).

automation are not likely to demonstrate massive reductions in their TAT performances. More likely than not, they will reap the benefits of sustainable and recurring good TAT performances, thereby rescinding the often-held inaccurate view of a huge TAT reduction from the implementation of laboratory automation.

Did laboratory automation reduce manpower needs? Over the last ten years, before and after we moved into the new premises, the workload has increased steadily (compound annual growth rate of $5 \%$ coupled with expansion of test repertoire) against a fairly constant headcount. Increased productivity has been realised through laboratory automation. The predicted staffing norm if there were no laboratory automation has not been exercised, as the prevailing staff number was able to maintain service standards (Fig. 4). Adoption of automation can and should take away mundane, repetitive and manual steps, thereby reducing errors and allowing redefinition of the job roles of skilled manpower towards value-added activities such as quality control and quality assurance, as well as facilitating expansion of the laboratory repertoire to include tests performed via state-of-the-art but labourintensive platforms, such as electrophoresis, high-performance liquid chromatography and tandem mass spectrometry.

Our test volume of 9.0 million (in 2016) would have been perceived to be a challenge to service delivery if the prediction of continued growth had been upheld without laboratory automation and/or increase in manpower. However, with the current configuration of the laboratory automation system and high-capacity, high-throughput chemistry analysers working in tandem with the processing workflow, double-digit (in millions) test volumes should still remain manageable in the next five years without the need to increase manpower. With constraints from the present talent pool and manpower resources, laboratory automation is likely to be a core feature in any medium-to-large clinical laboratory.

In terms of performance benchmarking, our productivity appears to be on par with that of the 98 US institutions surveyed in the CAP Q-Probe study in 2010: ${ }^{(17)}$ our statistic of 200,164 investigations per (clinical) FTE for clinical biochemistry alone (2015 data) compares favourably with the 117,616 billable test per (non-management) FTE for the US chemistry, haematology and microbiology laboratories combined. Our clinical laboratory's clinical-management FTE ratio of 17.1 also compares well with the US non-management/management ratio of 20.2.

With end-to-end laboratory automation, a blood specimen with its CPOE-ID label affixed travels to the clinical laboratory and directly enters the analyser or TLA. All test order information, patient demographics and admission details are traceable to the ordering location. Quality metrics on laboratory errors, previously observed with laboratory-based test requisition and specimen labelling, has become a thing of the past with endto-end laboratory automation. The clinical laboratory staff no longer handle test requisition and need only ensure that specimen processing and testing proceed smoothly. 


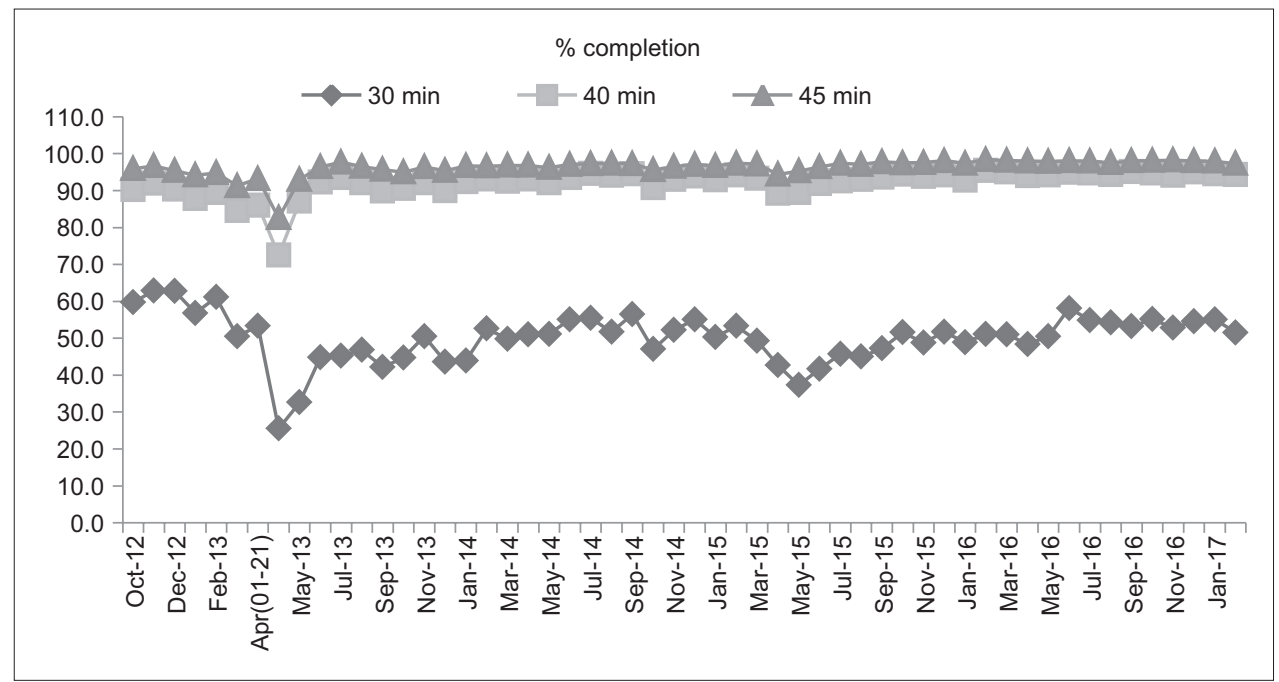

Fig. 3 Graph shows the monthly turnaround time for STAT requests from October 2012 to January 2017 (April 2013: relocation of laboratories to new facility; April 2015: MEDITECH laboratory information system upgrade).

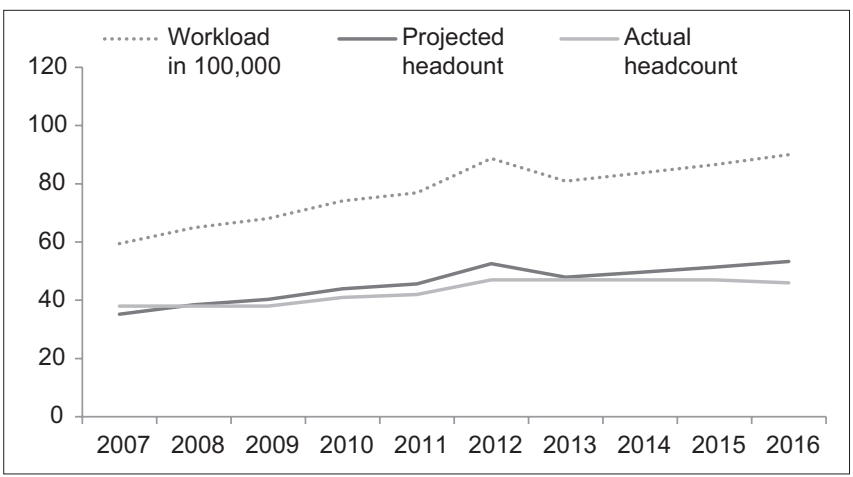

Fig. 4 Graph shows manpower status with actual headcount and projected headcount based on 2007-2009 staffing norm vs. test workload from 2007 to 2016 (2013: relocation to new facility).

Besides providing relief from the need to increase manpower, what other benefits justify the initial capital investment outlay? Beyond considering the efficiencies and effectiveness afforded by laboratory automation, positive impact on patient safety should also be a requirement rather than a caveat in a typical return of investment study, especially with the concept of patient safety receiving greater visibility and attention nowadays. Laboratory staff not only have to devote a substantial amount of time monitoring specimen processing and quality indicators, but are also expected to recover quickly from mechanical stops and error flags and have contingency measures in place should the specimen delivery system (the track) fail due to various reasons (e.g. power outage, routing mechanism faults). Informatics is central to optimal laboratory automation usage, much as the electronic medical record is now ubiquitous for effective management of a patient's medical condition. Automation enabled by mechanics and information technology (IT) will allow laboratory staff who are IT-savvy to navigate and manage information flow of the delivery, analytical and archival systems. One effective way to free up manpower time, especially with respect to result reporting, is to enable auto-validation of standard test results. Through intelligent use of auto-verification rules, about $80 \%$ of routine tests can be auto-validated and released to the patients' medical records. ${ }^{(18-20)}$ We have already taken small steps towards auto-validation with on-site glycated haemoglobin test results at polyclinics, ${ }^{(21)}$ which appears to be working well. It will be useful to extend the process to central laboratory tests.

\section{CONCLUSION}

In the frontier of clinical diagnostics technology, it is clear that cost management, manpower management and integration of systems are keys to enabling successful implementation. ${ }^{(13,14)}$ Automation aims to manage increasing workload demands, reduce errors and enhance laboratory performance while managing a limited labour force. Improving laboratory functions is not just about procuring equipment that is technologically sophisticated; instead, it requires an orchestrated approach that integrates best practices in lean work processes and IT support with skilled manpower. In this era of industry competition over a shrinking workforce, adoption of technology will certainly go a long way towards mitigating the challenges of these uncertain times.

\section{REFERENCES}

1. Castleberry BM, Wargelin L. 1992 wage and vacancy survey of US medical laboratory positions: part II. Lab Med 1993; 24:161-4.

2. Ward-Cook K, Tannar S. 2000 wage and vacancy survey of medical laboratories: wages on the upswing, vacancy rates accelerate. Lab Med 2001; 32:124-38.

3. Garcia E, Ali A, Choudhry S. The American Society for Clinical Pathology's 2012 vacancy survey of clinical laboratories in the United States. Lab Med 2013; 44:e1-18.

4. Badrick T. Is there a future for medical scientists and their associations? Aus J Med Sci 2007; 28:3-13.

5. Population.sg Team. Older Singaporeans to double by 2030. Available at: https://population.sg/articles/older-singaporeans-to-double-by-2030. Accessed April 20, 2017

6. Sasaki M, Kageoka T, Ogura K, et al. Total laboratory automation in Japan. Past, present, and the future. Clin Chim Acta 1998; 278:217-27.

7. Felder RA. The Clinical Chemist: Masahide Sasaki, MD, PhD (August 27, 1933-September 23, 2005). Clin Chem 2006; 52:791-2.

8. Sng EH. The science of medical detection: 100 years of pathology, 1903-2003. Singapore: Department of Pathology, Singapore General Hospital, 2003.

9. Ng WY, Yeo CP. Transforming the 24/7 clinical laboratories: design considerations, workflow and relocation to new diagnostics building. Int J Pathol Clin Res 2016; 2:024.

10. Henderson AR, Gardner MD. Clinical chemistry laboratory productivity: a comparison between a Canadian and a British teaching hospital. J Clin Pathol $1981 ; 34: 44-8$ 
11. Galloway M, Nadin L. Benchmarking and the laboratory. J Clin Pathol 2001 54:590-7.

12. Hernandez JS. Cost-effectiveness of laboratory testing. Arch Pathol Lab Med 2003; $127: 440-5$

13. Seaberg RS, Stallone RO, Statland BE. The role of total laboratory automation in a consolidated laboratory network. Clin Chem 2000; 46:751-6.

14. Streitberg GS, Bwititi PT, Angel L, Sikaris KA. Automation and expert systems in a core clinical chemistry laboratory. JALA 2009; 14:94-105.

15. Streitberg GS, Angel L, Sikaris KA, Bwititi PT. Automation in clinical biochemistry. Core, peripheral, STAT and specialist laboratories in Australia. JALA 2012; 17:387-94.

16. Zaninotto M, Plebani M. The "hospital central laboratory": automation, integration and clinical usefulness. Clin Chem Lab Med 2010; 48:911-7.

17. Jones BA, Darcy T, Souers RJ, Meier FA. Staffing benchmarks for clinical laboratories: a College of American Pathologists Q-Probes study of laboratory staffing at 98 Institutions. Arch Pathol Lab Med 2012; 136:140-7.

18. Krasowski MD, Davis SR, Drees D, et al. Autoverification in a core clinical chemistry laboratory at an academic medical center. J Pathol Inform 2014; $5: 13$.

19. The Norwegian Institute of Biomedical Science. Future trends in biomedical laboratory science: a Norwegian perspective. Available at: https://www. nito.no/contentassets/7152ab4936194074b7b10d18500bcfa7/future-trendsin-biomedical-laboratory-science---norwegian-perspective.pdf. Accessed April 20, 2017.

20. Foskett J. Making the most of autovalidation. In: Clinical Lab Products [online]. Available at: http://www.clpmag.com/2005/06/making-the-most-ofautovalidation/. Accessed April 18, 2017.

21. Lim K, Yeo CP. Abstract B018. Utilising information technology (IT) to improve work processes at the Satellite Laboratories. 66th AACC Annual Scientific Meeting and Clinical Expo, Chicago, USA, 27-31 July 2014. 